\title{
VIBRATION EFFECTS ON HEAT TRANSFER DURING SOLIDIFICATION OF PARAFFIN
}

\author{
Johnathan Vadasz *, Josua Meyer, Saneshan Govender, Gennady Ziskind \\ ${ }^{*}$ Author for correspondence \\ Department of Mechanical Engineering \\ University of Pretoria \\ Pretoria, GP, South Africa
}

\begin{abstract}
Previous work looked at the solidification process of PCM (phase change material) paraffin wax. Experimental results were compared with numerical work done in CFD package FLUENT. In the current study, the effects of vibration on heat transfer during the solidification process of PCM in a sphere shell are investigated. Enhancement of heat transfer results in quicker solidification times and desirable mechanical properties of the solid. The amount of PCM used was kept constant during each experiment by using a digital scale to check the weight, and thermocouple to check consistent temperature. A small amount of air was present in the sphere so that the sphere was not filled completely. Commercially available paraffin wax, RT35, was used in the experiments. Experimentations were done on a sphere of $40 \mathrm{~mm}$ diameter, wall temperature $20^{\circ} \mathrm{C}$ below mean solidification temperature, and consistent initial temperature. A vibration frequency was varied from $10-300 \mathrm{~Hz}$ was applied to the set-up and results compared with that of no vibration. Samples were taken at different times during the solidification process and compared with respect to solid material present.
\end{abstract}

\section{INTRODUCTION}

Directional solidification is a topic of wide interest due to its importance to the iron and steel industry. Solute convection in the solidification process results in channel formation, which has a freckle like appearance in the cross-section and has a critical effect on the mechanical strength of casting.

Rotational effects $[1,2,3]$ as well as gravitational effects $[4,5]$ on solidification are well documented and show a stabilizing effect on convection for the synchronous solutions, but slowly destabilizes convection for the region of sub-harmonic solutions [6]. Additional numerical results for convection in a porous layer subjected to vibration and heated from below, show that increasing the frequency of vibration causes the amplitude of convection to approach zero [7].

Experimental results using a hand tapping technique have shown an improvement in density, hardness, ultimate tensile strength, and \% elongation [8]. This has proven to be an inexpensive technique for improving the properties of long freezing range LM 25 or $356 \mathrm{Al}$ alloys.

Even though the hand tapping technique proves useful, it is unpractical for mass production. This method is also susceptible on an uneven vibration amplitude and frequency due to the human factor. Further experimentation is required to prove the necessity for implementing the vibration technique, and a better method is required to introduce vibrations.

\section{NOMENCLATURE}

\section{Latin Symbols}

$$
\begin{aligned}
& g_{*}=\text { acceleration due to gravity. } \\
& H_{*}=\text { the height of the layer. } \\
& L_{*}=\text { the length of the porous domain. } \\
& L=\text { reciprocal of aspect ratio, equals } L_{*} / H_{*} . \\
& R a=\text { Rayleigh number, equals } \beta_{*} \Delta T_{c} g_{*} H_{*} k_{*} M_{f} / \alpha_{e^{*}} \nu_{*} . \\
& P r_{\mathrm{D}}=\text { Darcy-Prandtl number equals } \oslash \mathrm{Pr} / \mathrm{Da} . \\
& \mathrm{R}=\text { scaled Rayleigh number } \\
& t=\text { time (dimensionless) } \\
& T=\text { dimensionless temperature, }\left(T_{*}-T_{C}\right) /\left(T_{H}-T_{C}\right) . \\
& \mathrm{T}_{\mathrm{C}}=\text { coldest wall temperature. } \\
& \mathrm{T}_{\mathrm{H}}=\text { hottest wall temperature. } \\
& \mathrm{X}=\text { rescaled amplitude. } \\
& \mathrm{Y}=\text { rescaled amplitude. } \\
& \mathrm{Z}=\text { rescaled amplitude. } \\
& \text { Greek Symbols } \\
& \varnothing=\text { porosity } \\
& \psi=\text { stream function. } \\
& \Delta T_{c}=\text { characteristic temperature difference. } \\
& \omega=\text { frequency. } \\
& \delta=\text { amplitude } \\
& \text { Subscripts } \\
& *=\text { dimensional values. } \\
& c r=\text { critical values. }
\end{aligned}
$$


$C=$ related to the coldest wall.

$H=$ related to the hottest wall.

\section{PROBLEM FORMULATION}

\section{Numerical:}

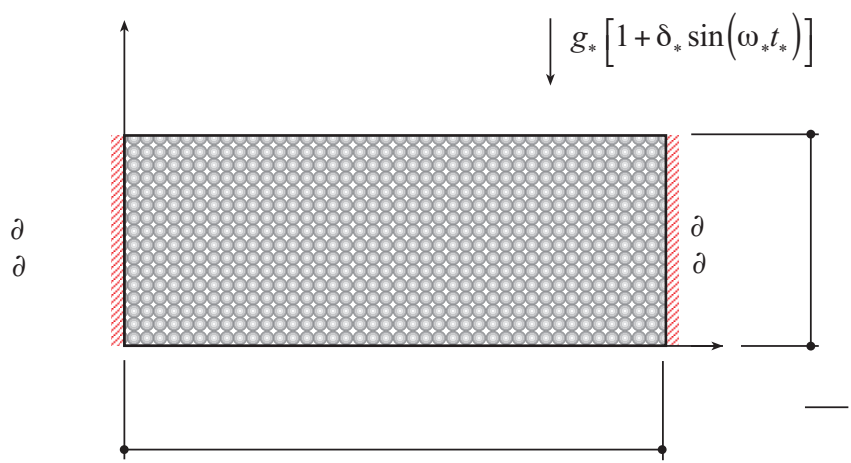

Figure 1: Porous media heated from below, subject to vibration.

The aspect ratio is defined by $L=\frac{L_{*}}{H_{*}}$

$\left[\frac{1}{P r_{D}} \frac{\partial}{\partial \tilde{t}}+1\right] \nabla^{2} \psi+R a[1+\delta \sin (\tilde{\omega} \tilde{t})] \frac{\partial T}{\partial x}=0$

$\frac{\partial T}{\partial t}+\frac{\partial \psi}{\partial z} \frac{\partial T}{\partial x}-\frac{\partial \psi}{\partial x} \frac{\partial T}{\partial z}=\nabla^{2} T$

We separate the stream function and temperature into a basic conduction part and variation convection one in the form

$\psi=\underbrace{\psi_{B}}_{=0}+\psi_{V}=\psi_{V}$

$T=\underbrace{T_{B}}_{=1-z}+T_{V}=1-z+T_{V}$

Substituting (2) into (1) yields

$\left[\frac{1}{P r_{D}} \frac{\partial}{\partial \tilde{t}}+1\right] \nabla^{2} \psi_{V}+R a[1+\delta \sin (\tilde{\omega} \tilde{t})] \frac{\partial T_{V}}{\partial x}=0$

$\frac{\partial T_{V}}{\partial t}+\frac{\partial \psi_{V}}{\partial z} \frac{\partial T_{V}}{\partial x}+\frac{\partial \psi_{V}}{\partial x}-\frac{\partial \psi_{V}}{\partial x} \frac{\partial T_{V}}{\partial z}=\nabla^{2} T_{V}$

We consider the truncated expansion

$\psi_{V}=A_{11} \sin \left(\frac{\pi x}{L}\right) \sin (\pi z)$

$T_{V}=B_{11} \cos \left(\frac{\pi x}{L}\right) \sin (\pi z)+B_{02} \sin (2 \pi z)$

This representation is equivalent to a Galerkin expansion of the solution in both the $x$ and $z$ directions, truncated when $(i+j)=2$, where $i$ is the Galerkin summation index in the $x$ direction while $j$ is the Galerkin summation index in the $z$ direction.

Then, from (4) we can perform the derivatives

$$
\begin{aligned}
\nabla^{2} \psi_{V}= & -\frac{\pi^{2}}{L^{2}} A_{11} \sin \left(\frac{\pi x}{L}\right) \sin (\pi z)-\pi^{2} A_{11} \sin \left(\frac{\pi x}{L}\right) \sin (\pi z)= \\
& -\pi^{2}\left(\frac{1}{L^{2}}+1\right) A_{11} \sin \left(\frac{\pi x}{L}\right) \sin (\pi z) \\
\frac{\partial \psi_{V}}{\partial z}= & \pi A_{11} \sin \left(\frac{\pi x}{L}\right) \cos (\pi z) \\
\frac{\partial \psi_{V}}{\partial x}= & \frac{\pi}{L} A_{11} \cos \left(\frac{\pi x}{L}\right) \sin (\pi z)
\end{aligned}
$$

$\frac{\partial T_{V}}{\partial x}=-\frac{\pi}{L} B_{11} \sin \left(\frac{\pi x}{L}\right) \sin (\pi z)$

$\frac{\partial T_{V}}{\partial z}=\pi B_{11} \cos \left(\frac{\pi x}{L}\right) \cos (\pi z)+2 \pi B_{02} \cos (2 \pi z)$

$$
\begin{aligned}
\nabla^{2} T_{V}=-\pi^{2}\left(\frac{1}{L^{2}}+1\right) B_{11} \cos \left(\frac{\pi x}{L}\right) & \sin (\pi z) \\
& -4 \pi^{2} B_{02} \sin (2 \pi z)
\end{aligned}
$$

Substituting (4) into equation ( $3 a$ ) by using the expressions for the derivatives from (5) yields

$$
\begin{aligned}
& -\left[\frac{1}{\operatorname{Pr}_{D}} \frac{d}{d \tilde{t}}+1\right]\left[\pi^{2}\left(\frac{1}{L^{2}}+1\right) A_{11} \sin \left(\frac{\pi x}{L}\right) \sin (\pi z)\right] \\
& -R a[1+\delta \sin (\tilde{\omega} \tilde{t})]\left[\frac{\pi}{L} B_{11}\right] \sin \left(\frac{\pi x}{L}\right) \sin (\pi z)=0
\end{aligned}
$$

which upon definition of $1 / \gamma=1 / L^{2}+1$ can be grouped in the form

$$
\begin{aligned}
\left\{\frac{\pi^{2}}{\gamma}\left[\frac{1}{P r_{D}} \frac{d}{d \tilde{t}}+1\right] A_{11}+\frac{\pi R a}{L}[1+\delta \sin (\tilde{\omega} \tilde{t})] B_{11}\right\} \\
\cdot \sin \left(\frac{\pi x}{L}\right) \sin (\pi z)=0
\end{aligned}
$$

or in the expanded form

$$
\begin{aligned}
\left\{\frac{\pi^{2}}{\gamma \operatorname{Pr}_{D}} \frac{d A_{11}}{d \tilde{t}}+\frac{\pi^{2}}{\gamma} A_{11}+\frac{\pi R a}{L}[1+\delta \sin (\tilde{\omega} \tilde{t})] B_{11}\right\} & \cdot \sin \left(\frac{\pi x}{L}\right) \sin (\pi z)=0
\end{aligned}
$$

where

$\frac{1}{\gamma}=\frac{1}{L^{2}}+1$ or $\gamma=\frac{L^{2}}{L^{2}+1}$ 
The nonlinear terms $\left(\partial \psi_{V} / \partial z\right)\left(\partial T_{V} / \partial x\right)$ and $\left(\partial \psi_{V} / \partial x\right)\left(\partial T_{V} / \partial z\right)$ in equation (3b) are evaluated from (5) in the form

$$
\begin{aligned}
\frac{\partial \psi_{V}}{\partial z} \frac{\partial T_{V}}{\partial x}=-\frac{\pi^{2}}{L} A_{11} B_{11} \sin ^{2}\left(\frac{\pi x}{L}\right) & \sin (\pi z) \cos (\pi z) \\
= & -\frac{\pi^{2}}{2 L} A_{11} B_{11} \sin ^{2}\left(\frac{\pi x}{L}\right) \sin (2 \pi z)
\end{aligned}
$$

and

$$
\begin{array}{r}
\frac{\partial \psi_{V}}{\partial x} \frac{\partial T_{V}}{\partial z}=\frac{\pi^{2}}{L} A_{11} B_{11} \cos ^{2}\left(\frac{\pi x}{L}\right) \sin (\pi z) \cos (\pi z) \\
+\frac{2 \pi^{2}}{L} A_{11} B_{02} \cos \left(\frac{\pi x}{L}\right) \sin (\pi z) \cos (2 \pi z)= \\
\frac{\pi^{2}}{2 L} A_{11} B_{11} \cos ^{2}\left(\frac{\pi x}{L}\right) \sin (2 \pi z) \\
+\frac{2 \pi^{2}}{L} A_{11} B_{02} \cos \left(\frac{\pi x}{L}\right) \frac{1}{2}[-\sin (\pi z)+\sin (3 \pi z)]= \\
\frac{\pi^{2}}{2 L} A_{11} B_{11} \cos ^{2}\left(\frac{\pi x}{L}\right) \sin (2 \pi z) \\
-\frac{\pi^{2}}{L} A_{11} B_{02} \cos \left(\frac{\pi x}{L}\right) \sin (\pi z) \\
+\frac{\pi^{2}}{L} A_{11} B_{02} \cos \left(\frac{\pi x}{L}\right) \sin (3 \pi z)
\end{array}
$$

Therefore, the form which appears in (3b) is

$$
\begin{gathered}
\frac{\partial \psi_{V}}{\partial z} \frac{\partial T_{V}}{\partial x}-\frac{\partial \psi_{V}}{\partial x} \frac{\partial T_{V}}{\partial z}=-\frac{\pi^{2}}{2 L} A_{11} B_{11} \sin ^{2}\left(\frac{\pi x}{L}\right) \sin (2 \pi z) \\
-\frac{\pi^{2}}{2 L} A_{11} B_{11} \cos ^{2}\left(\frac{\pi x}{L}\right) \sin (2 \pi z) \\
+\frac{\pi^{2}}{L} A_{11} B_{02} \cos \left(\frac{\pi x}{L}\right) \sin (\pi z) \\
-\frac{\pi^{2}}{2 L} A_{11} B_{11}[\underbrace{\left[\sin ^{2}\left(\frac{\pi x}{L}\right)+\cos ^{2}\left(\frac{\pi x}{L}\right)\right] \sin (2 \pi z)}_{=1} \\
+\frac{\pi^{2}}{L} A_{11} B_{02} \cos \left(\frac{\pi x}{L}\right) \sin (\pi z) \\
-\frac{\pi^{2}}{L} A_{11} B_{02} \cos \left(\frac{\pi x}{L}\right) \sin (3 \pi z)=
\end{gathered}
$$

Substituting now (7) and (5) into (3b) yields

$$
\begin{aligned}
& \overbrace{\frac{\mathrm{d} B_{11} \cos \left(\frac{\pi x}{L}\right) \sin (\pi z)}{\mathrm{d} \tilde{t}}+\overbrace{\frac{\mathrm{d} B_{02} \sin (2 \pi z)}{\mathrm{d} \tilde{t} \sin }}^{O}}^{O} \overbrace{\overbrace{\frac{\pi^{2}}{2 L} A_{11} B_{11} \sin (2 \pi z)}^{\pi_{\pi^{2}}} A_{11} B_{02} \cos \left(\frac{\pi x}{L}\right) \sin (\pi z)}^{A}- \\
& \overbrace{\frac{\pi^{2}}{L} A_{11} B_{02} \cos \left(\frac{\pi x}{L}\right) \sin (3 \pi z)}^{A}+\overbrace{\frac{\pi}{L} A_{11} \cos \left(\frac{\pi x}{L}\right) \sin (\pi z)}^{A}= \\
& -\overbrace{\frac{\pi^{2}}{\gamma} B_{11} \cos (\underbrace{A}_{\left.\frac{\pi x}{L}\right) \sin (\pi z)}-\overbrace{4 \pi^{2} B_{02} \sin (2 \pi z)}^{o}}^{A}
\end{aligned}
$$

Grouping likewise terms leads to

$$
\begin{array}{r}
{\left[\frac{\mathrm{d} B_{11}}{\mathrm{~d} \tilde{t}}+\frac{\pi^{2}}{L} A_{11} B_{02}+\frac{\pi}{L} A_{11}+\frac{\pi^{2}}{\gamma} B_{11}\right] \cos \left(\frac{\pi x}{L}\right) \sin (\pi z)} \\
+\left[\frac{\mathrm{d} B_{02}}{\mathrm{~d} \tilde{t}}-\frac{\pi^{2}}{2 L} A_{11} B_{11}+4 \pi^{2} B_{02}\right] \sin (2 \pi z) \\
-\frac{\pi^{2}}{L} A_{11} B_{02} \cos \left(\frac{\pi x}{L}\right) \sin (3 \pi z)=0
\end{array}
$$

Summarizing the resulting equations (6) and (8) in the form

$$
\begin{aligned}
&\left\{\frac{\pi^{2}}{\gamma P r_{D}} \frac{d A_{11}}{d \tilde{t}}+\frac{\pi^{2}}{\gamma} A_{11}+\frac{\pi R a}{L}[1+\right.\left.\delta \sin (\tilde{\omega} t)] B_{11}\right\} \\
& \cdot \sin \left(\frac{\pi x}{L}\right) \sin (\pi z)=0
\end{aligned}
$$$$
\left[\frac{\mathrm{d} B_{11}}{\mathrm{~d} \tilde{t}}+\frac{\pi^{2}}{L} A_{11} B_{02}+\frac{\pi}{L} A_{11}+\frac{\pi^{2}}{\gamma} B_{11}\right] \cos \left(\frac{\pi x}{L}\right) \sin (\pi z)
$$$$
+\left[\frac{\mathrm{d} B_{02}}{\mathrm{~d} \tilde{t}}-\frac{\pi^{2}}{2 L} A_{11} B_{11}+4 \pi^{2} B_{02}\right] \sin (2 \pi z)
$$$$
-\frac{\pi^{2}}{L} A_{11} B_{02} \cos \left(\frac{\pi x}{L}\right) \sin (3 \pi z)=0
$$

Multiplying now equation (9a) by the corresponding eigenfunctions and integrating over the domain, produces a system of ordinary differential equations for the amplitudes $A_{11}(\tilde{t}), B_{11}(\tilde{t}), B_{02}(\tilde{t})$ as follows. Multiplying equation (9) by $\sin (\pi x / L) \sin (\pi z)$ and integrating while using the orthogonality relationships results in

$$
\frac{L}{4}\left\{\frac{\pi^{2}}{\gamma \operatorname{Pr}_{D}} \frac{d A_{11}}{d \tilde{t}}+\frac{\pi^{2}}{\gamma} A_{11}+\frac{\pi R a}{L}[1+\delta \sin (\tilde{\omega} t)] B_{11}\right\}=0
$$

Multiplying equation (9b) by $\cos \left(\frac{\pi x}{L}\right) \sin (\pi z)$ and integrating over the domain produces 
$\frac{L}{4}\left[\frac{\mathrm{d} B_{11}}{\mathrm{~d} \tilde{t}}+\frac{\pi^{2}}{L} A_{11} B_{02}+\frac{\pi}{L} A_{11}+\frac{\pi^{2}}{\gamma} B_{11}\right]=0$

Multiplying equation (9b) by $\sin (2 \pi z)$ and integrating over the domain yields

$\frac{L}{2}\left[\frac{\mathrm{d} B_{02}}{\mathrm{~d} \tilde{t}}-\frac{\pi^{2}}{2 L} A_{11} B_{11}+4 \pi^{2} B_{02}\right]=0$

The resulting equations are

$$
\begin{aligned}
& \frac{L}{4}\left\{\frac{\pi^{2}}{\gamma \operatorname{Pr}_{D}} \frac{d A_{11}}{d \tilde{t}}+\frac{\pi^{2}}{\gamma} A_{11}+\frac{\pi R a}{L}[1+\delta \sin (\tilde{\omega} t)] B_{11}\right\}=0 \\
& \frac{L}{4}\left[\frac{\mathrm{d} B_{11}}{\mathrm{~d} \tilde{t}}+\frac{\pi^{2}}{L} A_{11} B_{02}+\frac{\pi}{L} A_{11}+\frac{\pi^{2}}{\gamma} B_{11}\right]=0 \\
& \frac{L}{2}\left[\frac{\mathrm{d} B_{02}}{\mathrm{~d} \tilde{t}}-\frac{\pi^{2}}{2 L} A_{11} B_{11}+4 \pi^{2} B_{02}\right]=0
\end{aligned}
$$

By introducing for convenience the following notation

$\gamma=\frac{L^{2}}{L^{2}+1} ; \quad \theta=\frac{L}{\gamma}=\frac{L^{2}+1}{L}$

and by rescaling the time variable in the form

$t=\frac{\pi^{2}}{\gamma} \tilde{t}$

one may present equations (13)-(15) after multiplying each one of them by $\gamma / \pi^{2}$ in the form

$\frac{\gamma}{\pi^{2}} \frac{d A_{11}}{d \tilde{t}}=-\frac{\operatorname{Pr}_{D} \gamma}{\pi^{2}}\left\{A_{11}+\frac{\gamma R a}{L \pi}[1+\delta \sin (\tilde{\omega} \tilde{t})] B_{11}\right\}$

$\frac{\gamma}{\pi^{2}} \frac{\mathrm{d} B_{11}}{\mathrm{~d} \tilde{t}}=-\frac{\gamma}{\pi L} A_{11}-B_{11}-\frac{\gamma}{L} A_{11} B_{02}$

$\frac{\gamma}{\pi^{2}} \frac{\mathrm{d} B_{02}}{\mathrm{~d} \tilde{t}}=-4 \gamma B_{02}+\frac{\gamma}{2 L} A_{11} B_{11}$

to yield

$\frac{L}{4}\left\{\frac{1}{P r_{D}} \frac{d A_{11}}{d \tilde{t}}+A_{11}+\frac{R a}{\pi \theta}\left[1+\delta \sin \left(\frac{\tilde{\omega} \tilde{t} \pi^{2}}{\gamma}\right)\right] B_{11}\right\}=0$

$\frac{L}{4}\left[\frac{\mathrm{d} B_{11}}{\mathrm{~d} \tilde{t}} \frac{L^{2}}{\pi^{2}\left(L^{2}+1\right)}+\frac{1}{\theta} A_{11} B_{02}+\frac{1}{\theta \pi} A_{11}+B_{11}\right]=0$

$\frac{L}{2}\left[\frac{\mathrm{d} B_{02}}{\mathrm{~d} \tilde{t}} \frac{L^{2}}{\pi^{2}\left(L^{2}+1\right)}-\frac{1}{2 \theta} A_{11} B_{11}+\frac{4 L^{2}}{L^{2}+1} B_{02}\right]=0$

where the forcing vibration's frequency was also rescaled in (18a) in the form

$\omega=\frac{\tilde{\omega} \gamma}{\pi^{2}}$

In the limit, when $\delta=0$, i.e. without vibrations the system has the following stationary (fixed) points $A_{11, f}=B_{11, f}=B_{02, f}=0$, the trivial motionless conduction solution that is expected to be stable for $R a<4 \pi^{2}$, and the following steady convective solution that is expected to be stable for $4 \pi^{2}<R a<R a_{0}$

$$
\begin{aligned}
& A_{11, f}=\mp \frac{2 \sqrt{2} \gamma^{1 / 2} R a^{1 / 2}}{\pi}\left[1-\frac{\pi^{2} \theta^{2}}{R a}\right]^{1 / 2} \\
& B_{11, f}= \pm \frac{2 \sqrt{2} L}{\gamma^{1 / 2} R a^{1 / 2}}\left[1-\frac{\pi^{2} \theta^{2}}{R a}\right]^{1 / 2} \\
& B_{02, f}=\frac{\pi \theta^{2}}{R a}-\frac{1}{\pi}=\frac{1}{\pi}\left[\frac{\pi^{2} \theta^{2}}{R a}-1\right]
\end{aligned}
$$

Rescaling the Rayleigh and Darcy Prandtl numbers in the form

$R=\frac{R a}{\pi^{2} \theta^{2}}, \alpha=\frac{\operatorname{Pr}_{D} \gamma}{\pi^{2}}$

yields

$$
\begin{aligned}
& A_{11, f}=\mp 2 \sqrt{2} \underbrace{\gamma^{1 / 2} \theta}_{=L / \gamma^{2}} R^{1 / 2}\left[1-\frac{1}{R}\right]^{1 / 2}=\mp 2 \sqrt{2} \gamma^{1 / 2} \theta(R-1)^{1 / 2} \\
& B_{11, f}= \pm \frac{2 \sqrt{2} \gamma^{1 / 2}}{\pi R^{1 / 2}}\left[1-\frac{1}{R}\right]^{1 / 2}= \pm \frac{2 \sqrt{2} \gamma^{1 / 2}}{\pi R^{1 / 2}}\left[\frac{R-1}{R}\right]^{1 / 2} \\
&= \pm \frac{2 \sqrt{2} \gamma^{1 / 2}}{\pi} \frac{(R-1)^{1 / 2}}{R}
\end{aligned}
$$

$B_{02, f}=\frac{1}{\pi}\left[\frac{1}{R}-1\right]=-\frac{(R-1)}{\pi R}$

A first rescaling of the amplitudes is convenient for plotting the bifurcation diagrams

$$
\begin{aligned}
& \tilde{A}_{11}=-\frac{A_{11}}{2 \sqrt{2} \gamma^{1 / 2} \theta} \rightarrow \tilde{A}_{11, f}= \pm(R-1)^{1 / 2} \\
& \tilde{B}_{11}=\frac{\pi B_{11}}{2 \sqrt{2} \gamma^{1 / 2}} \rightarrow \tilde{B}_{11, f}= \pm \frac{(R-1)^{1 / 2}}{R} \\
& \tilde{B}_{02}=-\pi B_{02} \rightarrow \tilde{B}_{02, f}=\frac{(R-1)}{R}
\end{aligned}
$$

Rescaling the amplitudes again in a way that removes the explicit dependence of the fixed points on $R$ in the form

$$
\begin{aligned}
& X=-\frac{A_{11}}{2 \sqrt{2} \gamma^{1 / 2} \theta(R-1)^{1 / 2}} \rightarrow A_{11}=-2[2 \gamma(R-1)]^{1 / 2} \theta X \\
& Y=\frac{\pi R B_{11}}{2 \sqrt{2} \gamma^{1 / 2}(R-1)^{1 / 2}} \rightarrow B_{11}=\frac{2[2 \gamma(R-1)]^{1 / 2}}{\pi R} Y
\end{aligned}
$$

$Z=-\frac{\pi R}{(R-1)} B_{02} \quad \rightarrow \quad B_{02}=-\frac{(R-1)}{\pi R} Z$

The convective fixed points are now $X_{f}= \pm 1, Y_{f}= \pm 1, Z_{f}=1$.

Substituting the rescaled amplitudes by using (25) into the equations (17) yields

$$
\frac{\mathrm{d} X}{\mathrm{~d} t}=-\frac{\operatorname{Pr}_{D} \gamma}{\pi^{2}}\{X-[1+\delta \sin (\omega t)] Y\}
$$

$\frac{\mathrm{d} Y}{\mathrm{~d} t}=-Y+R X-(R-1) X Z$

$\frac{\mathrm{d} Z}{\mathrm{~d} t}=4 \gamma(X Y-Z)$ 
To summarize, the rescaled equations have the form

$$
\begin{aligned}
& \dot{X}=-\alpha\{X-[1+\delta \sin (\omega t)] Y\} \\
& \dot{Y}=-Y+R X-(R-1) X Z \\
& \dot{Z}=4 \gamma(X Y-Z)
\end{aligned}
$$

where the Newtonian notation for time derivatives was introduced in the form

$$
\dot{X}=\frac{\mathrm{d} X}{\mathrm{~d} t}, \dot{Y}=\frac{\mathrm{d} Y}{\mathrm{~d} t}, \dot{Z}=\frac{\mathrm{d} Z}{\mathrm{~d} t}
$$

The controlling parameters are

$$
R=\frac{R a}{\pi^{2} \theta^{2}} ; \alpha=\frac{P r_{D} \gamma}{\pi^{2}} ; \gamma=\frac{L^{2}}{L^{2}+1} ; \omega=\frac{\tilde{\omega} \gamma}{\pi^{2}}
$$

and $\delta$ as the forcing vibration's amplitude.

Also, in (31) and (33) the following parameters and variables are defined

$$
\begin{aligned}
& \theta=\frac{L}{\gamma}=\frac{L^{2}+1}{L} \\
& t=\frac{\pi^{2}}{\gamma} \tilde{t} \\
& X=-\frac{A_{11}}{2 \sqrt{2} \gamma^{1 / 2} \theta(R-1)^{1 / 2}} \\
& Y=\frac{\pi R B_{11}}{2 \sqrt{2} \gamma^{1 / 2}(R-1)^{1 / 2}} \\
& Z=-\frac{\pi R}{(R-1)} B_{02}
\end{aligned}
$$

As $L \rightarrow \infty$ we obtain

$$
\gamma=\lim _{L \rightarrow \infty} \frac{L^{2}}{L^{2}+1}=\lim _{L \rightarrow \infty} \frac{1}{1+\frac{1}{L^{2}}}=1 \text { and }
$$

$\theta \rightarrow L \rightarrow \infty$ so $R \rightarrow 0$ for any finite value of $R a$. Therefore, for large values of $L$, i.e. $L>>1$ we get $\gamma \sim 1 ; \theta \sim L ; \alpha \sim \frac{P r_{D}}{\pi^{2}} ; t \sim \pi^{2} \tilde{t}$ and $R \sim \frac{R a}{\pi^{2}} L^{-2}$ as the approximations for $L \gg>1$. Large values of $L$ produce finite values of $R$ only for large values of $R a$. In order for $R=O(1)$ the Rayleigh number has to be of the order of magnitude $R a=O\left(L^{2}\right)$.

For the solution of the non-autonomous system (29), (30), (31) one introduces an additional dummy dependent variable

$$
W=\omega t
$$

transforming (29), (30), (31) into

$$
\begin{aligned}
& \dot{X}=-\alpha(X-Y)+\alpha \delta Y \sin (W) \\
& \dot{Y}=-Y+R X-(R-1) X Z \\
& \dot{Z}=4 \gamma(X Y-Z) \\
& \dot{W}=\omega
\end{aligned}
$$

\section{Experimental:}

Various samples were taken under different conditions to check the effect of vibration on the following: average density, void formation and solidification time.

To simplify the experimental procedure and reduce cost, solidification of Paraffin is used. Although this is not a metal the effects of vibration apply the same theory. This will give a good basis and will help with the understanding of the effects.

Due to the lower melting temperature $\left(35^{\circ} \mathrm{C}\right)$ of Paraffin, a furnace is not needed. Working at lower temperatures allows for simpler, more cost effective experiments to be run.

Sample spheres were prepared by drilling a $4 \mathrm{~mm}$ hole, then attaching a plastic pipe over the hole by means of hot glue and glue gun. The plastic pipe allowed the sphere to be completely submerged in the thermal bath. By submerging the ball completely in the water, a more uniform wall temperature is achieved. The PCM was heated and temperature constantly checked, until desired temperature is reached. The PCM was extracted using a syringe and inserted slowly into the sphere. Using a digital scale the weight of the PCM was measured to achieve consistency. The sphere was then secured in the vibration rig and lowered into the thermal bath. For experiments with no vibration the vibration source was omitted. The thermal bath was set to a constant temperature prior to starting the experiments and allowed to stabilize. The time for complete solidification was known from prior testing. Different samples were done for different time intervals and different frequencies. The sphere was taken out the vibration rig, plastic pipe was removed and the sphere cut in half. All samples were photographed extensively for comparison. Measurements were also taken of the PCM thickness at the walls and void size for comparison. Each sample was repeated under identical conditions to check for consistency of experimental procedure.

\section{RESULTS AND DISCUSSION}

Samples in figures 2(a) and 1(b) show the solid PCM were taken out after 10 minutes. It can be seen that more solid PCM was formed when the sample was excited by the vibration source; Measurements of wall thickness and average density taken support this. 


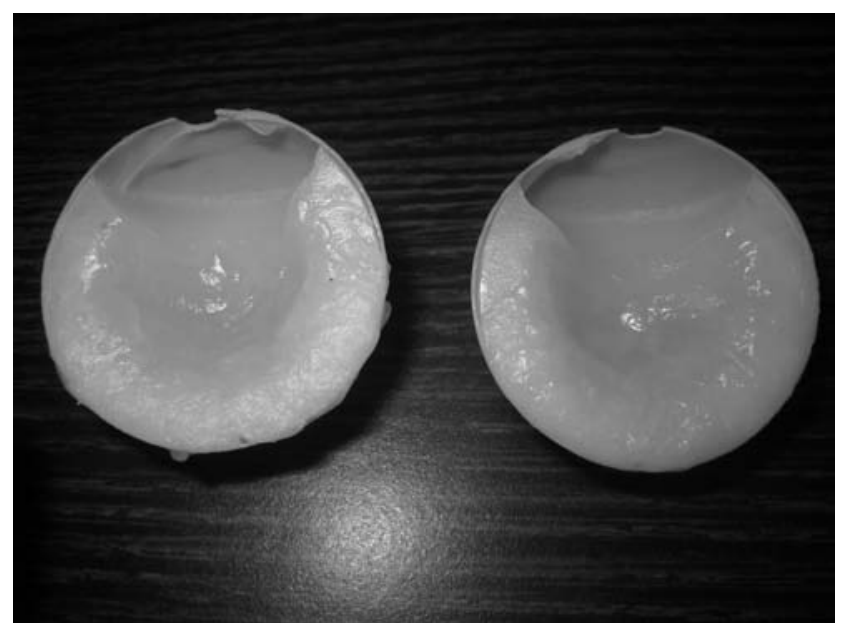

Figure 2(a): Cross section of the $40 \mathrm{~mm}$ ball starting with $\Delta \mathrm{T}=$ $20^{\circ}$ using RT35 paraffin wax, and allowing for 10 minutes of solidification time.

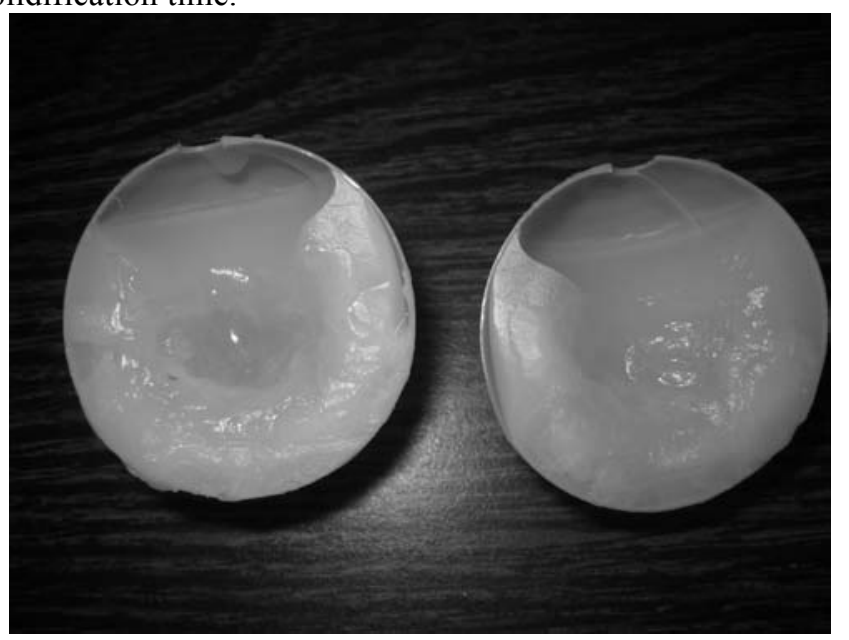

Figure 2(b): Cross section of the $40 \mathrm{~mm}$ ball starting with $\Delta \mathrm{T}=$ $20^{\circ}$ using RT35 paraffin wax, and allowing for 10 minutes of solidification time under a vibration frequesncy of $100 \mathrm{~Hz}$.

Samples in figures 3(a) and 3(b) show the solid PCM were fully solidified under $25 \mathrm{~Hz}$ and $10 \mathrm{~Hz}$ vibration frequency respectively. Apart from seeing a large effect on the void shape and size, the average density has also been affected. It can be seen tin figure $3(\mathrm{~b})$ that the material formed has formed a more porous structure (apart from the area near the wall). Figure 3(a) shows a more uniform solid structure. Other samples taken also showed different types of pores and average densities by changing the vibration frequency. Figure 3(c) shows the affect on the pores very well. Not only are there very small pours only seen under magnification, but there can be seen a very interesting arrangement of larger pores in the shape of a face.

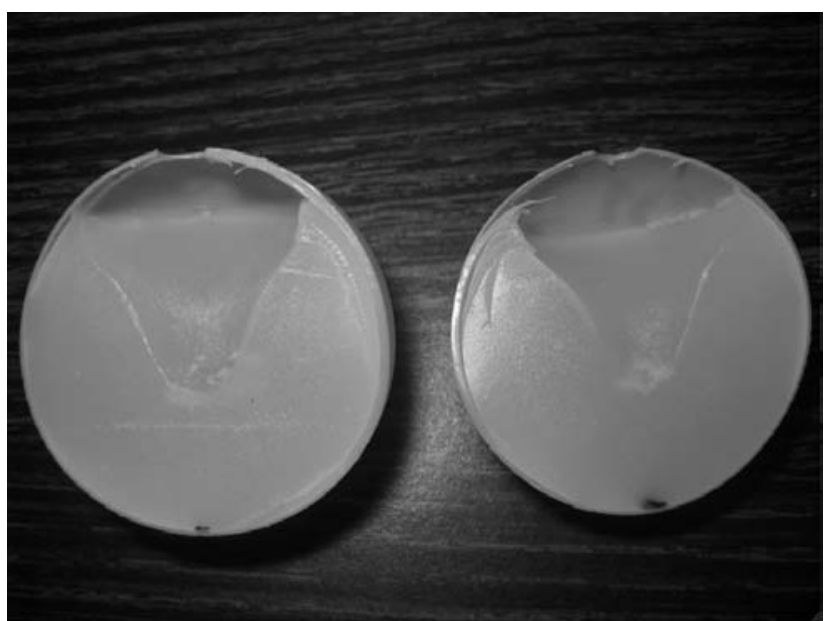

Figure 3(a): Cross section of the $40 \mathrm{~mm}$ ball starting with $\Delta \mathrm{T}=$ $20^{\circ}$ using RT35 paraffin wax, and allowing for full solidification under a vibration frequesncy of $25 \mathrm{~Hz}$.

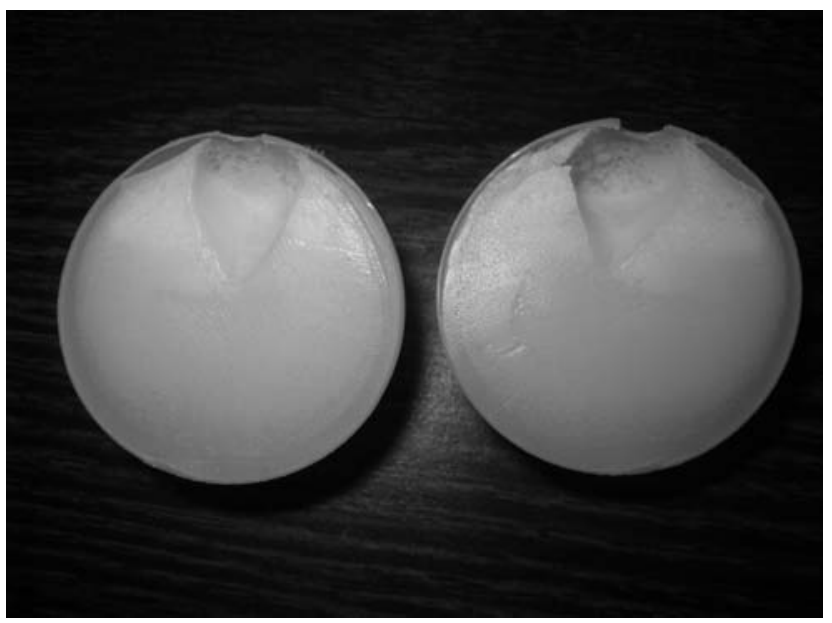

Figure 3(b): Cross section of the $40 \mathrm{~mm}$ ball starting with $\Delta \mathrm{T}=$ $20^{\circ}$ using RT35 paraffin wax, and allowing for full solidification under a vibration frequesncy of $10 \mathrm{~Hz}$.

After the samples were taken out the thermal bath and processed the weight was measured and recorded on a digital scale. The results seen in figure 2 are shown in Table 1. It can be seen that at 5 minutes there is significantly more solid formed under vibration comparing the $18.22 \mathrm{~g}$ to the $15.58 \mathrm{~g}$ under no vibration. The final time for solidification under no vibration is 30 minutes whereas under a vibration frequency of $100 \mathrm{~Hz}$ the time is 25 minutes.

Table 1: Measured weight of PCM at different times for no vibration and vibration at frequency of $100 \mathrm{~Hz}$.

\begin{tabular}{|l|l|l|}
\hline Time (minutes) & $\begin{array}{l}\text { 0 Hz (no vibration) } \\
\text { Weight of PCM } \\
\text { (grams) }\end{array}$ & $\begin{array}{l}\text { 100 Hz Weight of } \\
\text { PCM (grams) }\end{array}$ \\
\hline 5 & 15.58 & 18.22 \\
\hline 10 & 17.83 & 19.02 \\
\hline 20 & 19.80 & 20.21 \\
\hline 25 & NA & 21 \\
\hline 30 & 21.29 & NA \\
\hline
\end{tabular}


As can be seen in figure 4 there is more solid PCM formed at each point in time until final solidification time. The difference between the two final values of $0.29 \mathrm{~g}$ is due to initial amount of liquid PCM being different by the same amount. At 5 minutes there is a large difference between the two graphs and this difference decays with time, however never reaches zero. This means there is always more solid PCM formed under vibration then without. The reason for this may be due to the decay in effects of vibration due to more solid being formed.

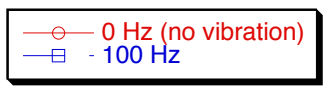

$\mathrm{RT} 35$ at $\Delta \mathrm{T}=\mathbf{2 0}$

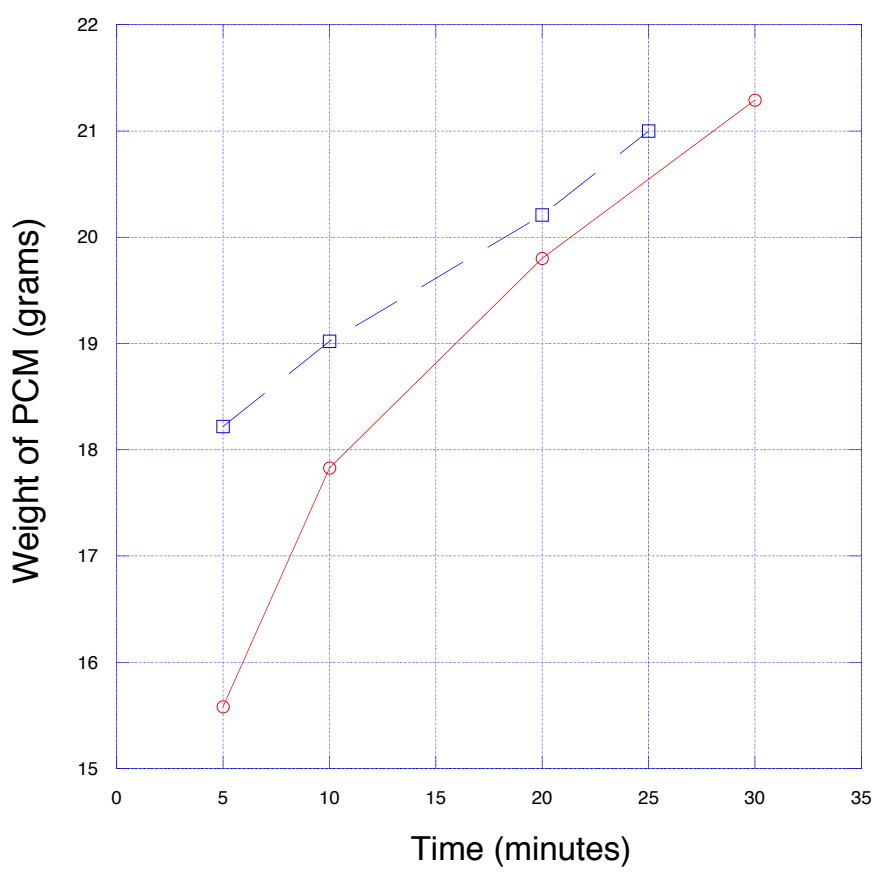

Figure 4: Weight of PCM formed versus time for no vibration and vibration frequency of $100 \mathrm{~Hz}$.

Measurements were also taken of the thickness of the solid PCM for comparison. These measurements validated the visual findings that there was more solid formed for the sample under vibration. Average density was also checked to see that the effects of vibration do not cause some kind of porous structure. It was seen that the average densities between the samples with and without vibration were similar. In fact the sample with vibration shows slightly higher density than that without. Doing this test shows there is more heat transfer with the vibration induced, and an overall 17\% improvement in solidification time calculated using equation 4.

The computed numerical results of amplitude vs. amplitude and vs. $t$ for a vibrating porous media heated from below at rescaled Rayleigh number $\mathrm{R}=25$, are presented in the next Figures.

Figure 5 shows a well known result for the chaotic behaiviour at $R=25$. This can be seen clearly in all the figures $5(\mathrm{a})-(\mathrm{d})$.
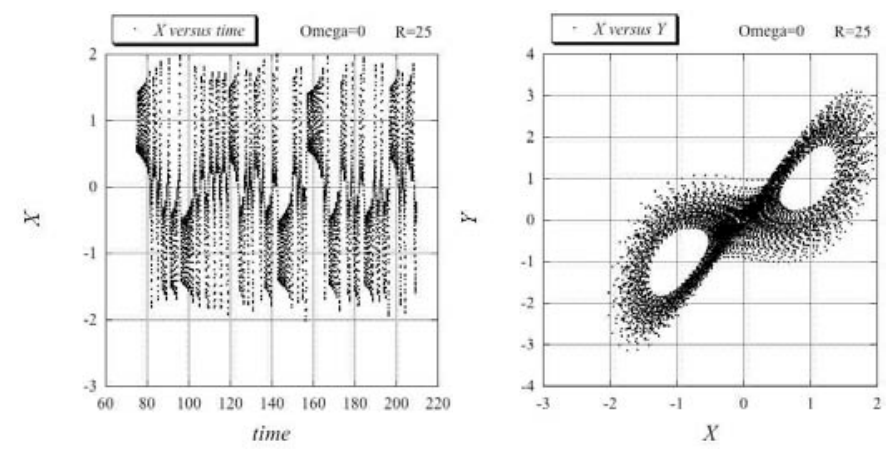

(a)

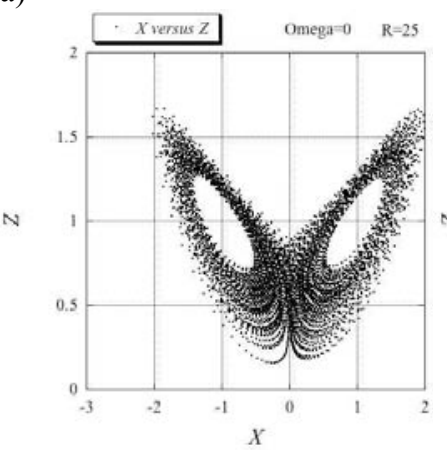

(c)

Figure 5: (a) Amplitude $X$ versus time under no vibration with rescaled Rayleigh number $R=25$. (b) Amplitude $X$ versus $Y$ under no vibration with rescaled Rayleigh number $R=25$. (c) Amplitude $X$ versus $Z$ under no vibration with rescaled Rayleigh number $R=25$. (d) Amplitude $Y$ versus $Z$ under no vibration with rescaled Rayleigh number $R=25$.

Figure 6(a) shows what seems to be a periodic function. Figures 6(b)-(d) further indicate a periodic relation with the projection of the amplitudes versus eachother. At an rescaled frequency omega value of 8 , the vibration has eliminated the chaotic behaiviour previously seen at a Rayleigh number of 25 . 


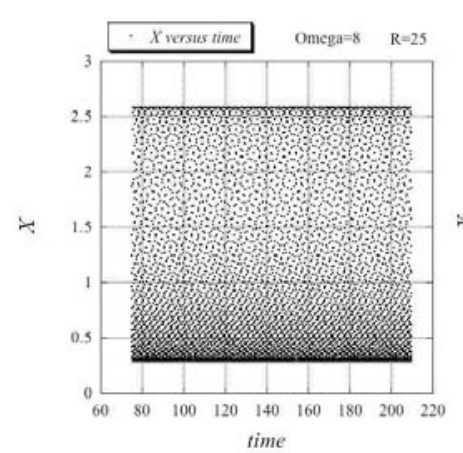

(a)

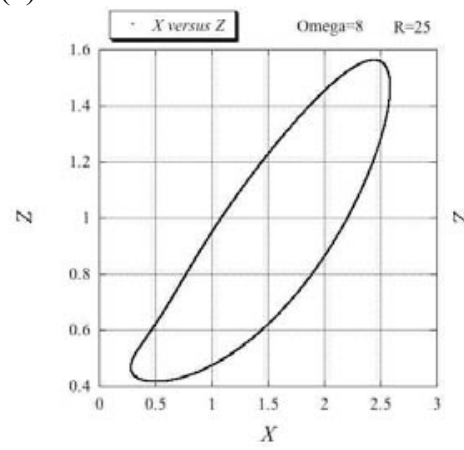

(c)

Figure 6: (a) Amplitude $X$ versus time under rescaled vibration frequency omega $=8$, with rescaled Rayleigh number $R=25$. (b) Amplitude $X$ versus $Y$ under rescaled vibration frequency omega $=8$, with rescaled Rayleigh number $R=25$. (c) Amplitude $X$ versus $Z$ under rescaled vibration frequency omega $=8$, with rescaled Rayleigh number $R=25$. (d) Amplitude $Y$ versus $Z$ under rescaled vibration frequency omega $=8$, with rescaled Rayleigh number $R=25$.

Figures 7 (a)-(d) show the result with a rescaled frequency omega of 7. In figure 7(a) it shows what seems to be chaotic behaiviour that transformes into periodic with time. Figures 6 (b)-(d) show a similar effect, where it looks as if there is both the periodic lines of the projected frequencies and some chaotic behaiviour.

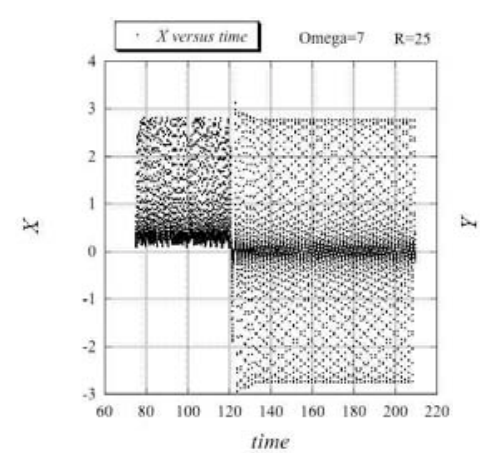

(a)

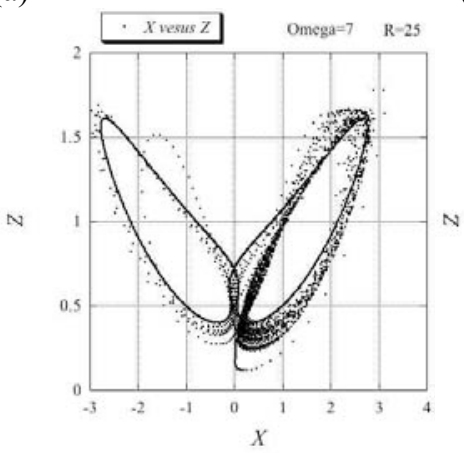

(b)
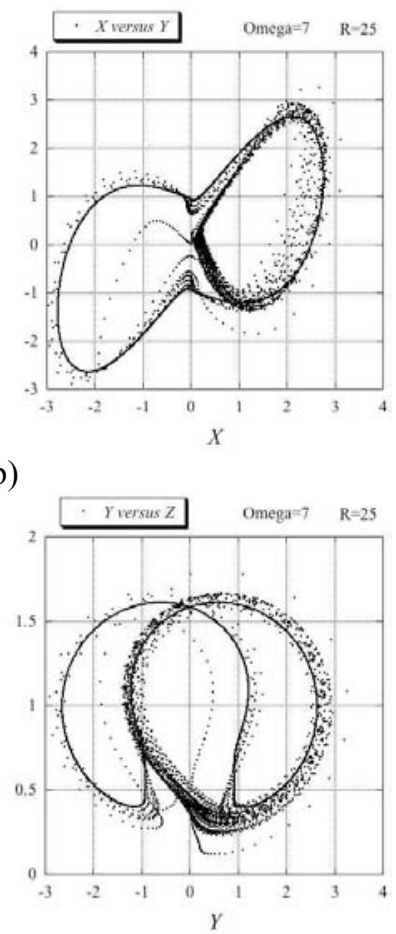

(d)

(c)

Figure 7: (a) Amplitude $X$ versus time under rescaled vibration frequency omega $=7$, with rescaled Rayleigh number $R=25$. (b) Amplitude $X$ versus $Y$ under rescaled vibration frequency omega $=7$, with rescaled Rayleigh number $R=25$. (c) Amplitude $X$ versus $Z$ under rescaled vibration frequency omega $=7$, with rescaled Rayleigh number $R=25$. (d) Amplitude $Y$ versus $Z$ under rescaled vibration frequency omega $=7$, with rescaled Rayleigh number $R=25$.

From figures 5-7 it can be seen that vibration has a large effect on the stability of the system. At certain frequencies the system turns from chaotic to periodic and back again.

\section{CONCLUSIONS}

Experimental results show how vibration is used to affect solidification time, average density, and void formation. Further work will need to be done to find a unique relationship between vibration and the above mentioned properties. Numerical results show how vibration can control the periodic or chaotic region in a porous media heated from below. Although the control system has not been identified, further work is justified with the results presented. It is important to mention that the experiments and numerical work are not directly comparable because of the different systems. The experiment is a solidification model and the numerical a porous media model. Some links can however be made; the anticipated affect of the vibration in the solidifcation model is in the porous region (solid/liquid interface), which would suggest further numerical and analytical work is needed with a solidification model. Also seeing that there is a clear affect on both experimental and numerical systems due to vibration, leads to the need for further work in this field. Emphasis is placed on the control of both systems by means of vibration. 


\section{REFERENCES}

[1] Govender, S., Vadasz, P., Moderate time scale linear stability of moderate Stefan number convection in rotating mushy layers, Journal of Porous Media, Vol. 5 (2), 2002, pp. 113-121.

[2] Govender, S., Vadasz, P., Weak Non-linear Analysis of Moderate Stefan Number Oscillatory Convection in Rotating Mushy Layers, Transport in Porous Media, Vol. 48 (3), 2002, pp. 353-372.

[3] Govender, S., Vadasz, P., Weak Non-linear Analysis of Moderate Stefan Number Stationary Convection in Rotating Mushy Layers", Transport in Porous Media, Vol. 49 (3), 2002, pp. 247-263.

[4] Amberg, G., Homsy, G.M., Nonlinear analysis of buoyant convection in binary solidification to channel formation, $J$. Fluid Mechanics, Vol. 252, 1993, pp.79-98.

[5] Anderson D.M., Worster, M.G., Weak nonlinear analysis of convection in mushy layers during the solidification of binary alloys, J. Fluid Mechanics, Vol. 302, 1995, pp. 307-331.

[6] Anderson D.M., Worster, M.G., A new oscillatory instability in mushy layers during the solidification of binary alloys, J. Fluid Mechanics, Vol. 307, 1996, pp. 245-267.

[7] S. Govender, Stability analysis of a porous layer heated from below and subjected to low frequency vibration: Frozen time analysis, Transport in Porous Media, Vol. 59, 2005, pp. 239-247.

[8] R. M. Pillai, K.S. Biju Kumar, B.C. Pai, A simple inexpensive technique for enhancing density and mechanical properties of Al-Si alloys, J. Materials Processing Technology, Vol. 146, 2004, pp.338-348. 